\title{
Onset of nanotube decay under extreme thermal and electronic excitations *
}

\author{
Yoshiyuki Miyamoto ${ }^{a}, \star \star$ \\ Savas Berber $^{b}$, Mina Yoon $^{b}$, Angel Rubio $^{c}$ and David Tománek ${ }^{b}$ \\ ${ }^{a}$ Fundamental Research Laboratories, NEC Corporation, \\ 34 Miyukigaoka Tsukuba, 305-8501 JAPAN \\ ${ }^{b}$ Department of Physics and Astronomy, \\ Michigan State University, East Lansing, Michigan 48824-1116, USA \\ ${ }^{c}$ Dpto. Fisica de Materiales, Facultad de Quimicas \\ U. Pais Vasco, Centro Mixto CSIC-UPV/EHU \\ Donostia International Physics Center (DIPC) \\ Apdo. 1072, 20018 San Sebastian/Donostia. SPAIN
}

\begin{abstract}
Stability test of nanotubes with presence of single vacancies have been performed by means of Tight-Binding molecular dynamics and of electron-ion dynamics within the framework of the density functional theory. A $4 \stackrel{\circ}{A}$ diameter nanotube having single vacancy with three dangling bonds has been found to retain its cylindrical shape under high temperature around $4000 \mathrm{~K}$, despite its large internal strain energy. Meanwhile, an electronic excitation of vacancy related state has shown considerable atomic displacement, which may cause extraordinary large lattice vibration or nanotube decay. Furthermore, the single vacancy can stabilize itself by making carbon dimer remaining only one dangling bond. Narrower nanotubes tend to prefer this self-stabilization and thus could be tolerant to presence of defects.
\end{abstract}

Key words: Carbon nanotube; Defect; Molecular dynamics; Density Functional Theory

PACS numbers: 61.48.+c, 61.72.Bb, and 71.15.Pd

\footnotetext{
$\bar{\star}$ Part of the present calculations were performed by using the SX4 Supercomputer system at the NEC Tsukuba Laboratories.

${ }^{\star \star}$ FAX: +81-298-50-2647, Email: y-miyamoto@ce.jp.nec.com (Y. Miyamoto)
}

Preprint submitted to Elsevier Science $\quad 29$ September 2001 


\section{Introduction}

After the discovery of carbon nanotube (CNT)[1], application to CNTelectronic devices has become one of main subjects, which could alternate to the conventional semiconductor technology. Yet many hurdles remain before achieving the real CNT-devices in order to go beyond Si-LSI. The Si-LSI technology established the standard criteria so-called as 'ten-nine' crystalline perfection, which guarantees reliability of Si-made devices. However, it is not well known whether such an unprecedented perfection is necessary for CNT-based technology. In this context, defects on CNT should be investigated intensively. Experimental study with use of the electron spin resonance technique[2,3] suggested the presence of the localized spin, which may relate with the dangling bonds. However, the illustrative information regarding atomic relaxation near the defected site still remains to be clarified.

Theoretically a simple model for the single vacancy is considered to be just a geometry obtained by removing one carbon atom from the honeycomb network. Previous theoretical work within tight-binding method treating the $\pi$ electrons[4] showed the influence of such kind of defects on the conductivity can be decreased with increasing tube circumference. Meanwhile, first-principles calculations [5] showed importance of treating $\sigma$ electrons in addition to the $\pi$ electrons in computing the I-V characteristics of CNTs with presence of vacancies. The $\sigma$ electrons of dangling bonds of the vacancy cause sharp dips in the I-V curves, while the $\pi$ electrons cause a broad dip. Except particular ranges of applied bias, which relates the local density of states near the defect, I-V curve of the defected CNT coincides with that of the perfect CNT. These theoretical works show that applying CNTs as parts of electronic device is feasible since even defected CNTs behave as perfect tube under tuned range of the applied biases or under the controlled density of defects. However, no attention has been paid on the stability of such defected CNTs during the device-run. In cases of application of CNTs to high-speed logic circuits[6], the stability is very crucial for the reliability of the devices since applying external bias to the CNT-device should be repeated more than million times per second throughout the usage for many days.

In this paper, we show our theoretical investigation of influence of single vacancies on CNT stability. We especially focus on narrow CNTs since the stability of highly curved narrow CNTs can be a benchmark to the stability of ordinary CNTs. We have performed the tight-binding molecular dynamics (TBMD) simulation and the time-dependent electron-ion dynamics within the density functional theory (DFT), in order to investigate the dynamics of CNTs with presence of single vacancies under thermal and electronic excitations. We first show considerable stability of a $(3,3)$ nanotube possessing single vacancies by performing the TBMD under high temperature up to 4000K. Recent experi- 
mental reports $[7,8]$ show existence of this very thin (4 $\stackrel{A}{ }$ diameter) CNT. We next demonstrate that an optical excitation induces lattice motion, in which kinetic energy concentrates to an atom adjacent to the vacancy. This athermal ionic motion might be an onset of a collapse of a CNT. Finally, we show self-stabilization of the single vacancy appeared in narrower CNTs, which reduces the number of dangling bonds. We expect that the final result of the self-stabilization is a promising sign of reliability of CNTs as part of electronic devices. In the following sections, we show our computational methods briefly and the details of our computational results. Finally, our concluding remarks will be given.

\section{Computational methods}

The stable geometry of the defected CNTs was determined by the total-energy band-structure calculations [9] within a framework of the local density approximation (LDA) of the DFT with use of pseudopotentials. Perdew-Zunger functional[10] fitted to the Ceperley-Alder calculations[11] was used to express exchange-correlation potential while the soft pseudopotentials[12] in the separable form[13] were used to express interactions between ions and valence electrons. In the LDA calculations, the plane wave basis set with kinetic cutoff energy of $40 \mathrm{Ry}$ was used to extending the valence Bloch states. The tubes are treated by repeating hexagonal cell keeping inter-tube distance more than $4 \stackrel{\circ}{ }$ and the period along with the tube axis was kept as triple or quadruple as the original one of the armchair CNT. The $\Gamma$ point was used for the momentum space integration.

In performing the TBMD simulations under thermal excitations, the Tight Binding total energy parameters developed in a former work[14] and the NosèHoover thermostat were adopted. The time-step for the simulation was 0.5 fs. We also performed molecular dynamics (MD) under electronic excitations on the basis of the DFT. To mimic the excited states, single electron was promoted from one occupied state in the valence bands to one unoccupied state in the conduction bands. The self-consistent solution of the Kohn-Sham equation under this promotion was set as the initial condition of the electronion dynamics. The molecular dynamics simulation under the electronic excited state should be proceed not by solving the secular equation for electronic wave functions but by solving the time-dependent Schrödinger equation. The excited states has finite lifetimes and sometime level alternation among the state with different occupation numbers occurs throughout the simulation. In the conventional $a b$ initio MD simulations, these two effects can easily be missed. That is why we must solve the time-dependent Scrödinger equation. In solving the time-dependent Scrödinger equation, the time-step should be much shorter than that of the MD as mentioned above. (The present time step is 
$1.9 \times 10^{-3}$ fs.) We applied the electron-ion dynamics within the LDA by solving the time-dependent Schrödinger equation (the time-dependent Kohn-Sham equation)[15], where the Suzuki-Trotter split operator method[16] was applied for the time evolution of electron wave functions, and Hellmann-Feynman forces on atoms were used to solve Newton equation of motion unless the simulation leaves from the adiabatic potential energy surface.

\section{Single vacancy having three dangling bonds.}

In this section, we present our computed results for CNTs with single vacancies. Figure 1 shows relaxed geometry of the $(n, n)$ nanotubes $(n=3 \sim 7)$ with presence of single vacancies. Similar to former theoretical works[4,5], each of three carbon atoms adjacent to a vacancy has one dangling bond and is coordinated by two other atoms apart from a vacancy. These structures were determined by the total-energy calculation[9] within the LDA under the periodic boundary conditions as mentioned before. The $\mathrm{C}-\mathrm{C}$ bond lengths in the two-hold coordinated carbon atoms are shorter than the regular C-C bond length by 0.03 to $0.04 \AA$. Note that the system has mirror symmetry with the mirror plane perpendicular to the tube axis, which crosses one of the three atoms adjacent to the vacancy.

\subsection{Stability test under high temperatures}

By applying the TBMD simulation, we have investigated the stability of narrow CNTs with presence of single vacancies. We chose $(3,3)$ and $(4,4)$ nanotubes each of which has a single vacancy at every triple period along with the tube axis. Then we start the MD simulation in which the temperature is raised by 400 degrees at every $0.75 \mathrm{ps}$ starting from an initial temperature of $400 \mathrm{~K}$. Figures 2(a) show the history of the temperature throughout the MD simulation for the case of the defected $(3,3)$ nanotube. The final temperature is around $4000 \mathrm{~K}$ but this includes considerable fluctuation on the time axis. The $(3,3)$ nanotube survived throughout the simulation. Figure 2 (b) shows a final snap shot of the simulation, in which the cylindrical form still remains. (We expect unzipping of this CNT under conditions of higher temperatures.)

Suppose the CNT plays a part of nano-electronic devices, the temperature should not be raised as much as the present TBMD simulation. We therefore conclude that CNTs with single vacancies do not decay due to the thermal effect throughout the usage of CNT devices. We also observed the survival of the defected $(4,4)$ nanotube and expect the survival of CNTs with larger diameters, because of smaller internal strain energy compared to that of the 
$(3,3)$ nanotube.

\subsection{Stability test under electronic excitations}

We also investigated the influence of the electronic excitation induced by illumination of the light. The shining of the light with low energy (from visible to infrared region) is ubiquitous and thus has to be considered throughout the device-run. We first analyzed the electronic structure of the single vacancy of $(3,3)$ nanotube. Figure 3 shows energy level of the $(3,3)$ nanotube near the Fermi level $\left(\mathrm{E}_{\mathrm{F}}\right)$ with a single vacancy at every quadruple cell of the armchair CNT. The geometry optimization has been done under the electronic ground state. The bars in the left column and panels connected with these bars by arrows are energy levels and charge contour maps of the states localized around the vacancy. According to the symmetry analysis of the localized states, the highest occupied (HO) state and lowest unoccupied (LU) state have symmetric characteristics, while the second $\mathrm{HO}$ and second LU state have asymmetric characteristics with respect to the mirror plane. Therefore, according to the optical selection rule, excitation from second $\mathrm{HO}$ to $\mathrm{LU}$ states or from $\mathrm{HO}$ to second LU states is allowed when optical dipole moment is parallel to the tube axis.

Next we explored an electronic excitation, which gives considerable driving forces on carbon atoms. When all atoms remain at their equilibrium positions under electronic ground state, an excitation from $\mathrm{HO}$ to second LU (a thick arrow in Fig. 3) gives considerable strength of forces of 0.02 Hartree/(Bohr radius) to two of the three atoms adjacent to the vacancy. These two atoms are labeled as $\mathrm{C}_{1}$ and $\mathrm{C}_{2}$ in Fig. 5, which will appear later. Upon promoting single electron from $\mathrm{HO}$ to second $\mathrm{LU}$ states, the level alternation occurred between the $\mathrm{HO}$ and second $\mathrm{HO}$ states and resulted in the energy levels shown in bars in the right column of Fig. 3. From the charge contour maps connected with these bars, we can identify the original eigen states. This level alternation is understood by a fact that original $\mathrm{HO}$ state is very localized as to change its eigenvalues by reducing its occupation number, and furthermore has different irreducible representation from that of the second $\mathrm{HO}$ state under the mirror symmetry of the system. Without taking the Frank-Condon relaxation, the excitation energy is obtained as $0.9 \mathrm{eV}$ within a LDA level of accuracy.

We then started the electron-ion dynamics to investigate the influence of this electronic excitation on the structural stability. So far, we have achieved $70 \mathrm{fs}$ in simulation time in which we can see the level alternation among the state with different occupation numbers. Figure 4 show time-evolution of the expectation values of the Kohn-Sham Hamiltonian, which can be interpreted as single-electron energy levels since the simulation moves on adiabatic potential 
energy surface, so far. The electron-hole pair created by the excitation became to be a pair of $\mathrm{HO}$ and LU states later than 15 fs. Within this simulation time, we have not seen the offset of the nonradiative decay of the excitation. Atomic geometry before and after the simulation, see Fig. 5 (a), shows a slight closure of the vacancy: The geometry at $70 \mathrm{fs}$ shows remarkable replacement of an atom labeled by $\mathrm{C}_{3}$, while other two atoms adjacent to the vacancy (labeled as $\mathrm{C}_{1}$ and $\mathrm{C}_{2}$ ) do not show a large deviation from their original locations. The trajectory of $\mathrm{C}_{1}$ and $\mathrm{C}_{2}$ atoms showed opening of the vacancy at first and then these two atoms change the directions toward their initial positions. Meanwhile, the $\mathrm{C}_{3}$ atom is highly accelerated so as to go inside the vacancy. Figure 5 (b) shows distribution of the kinetic energy on all atoms in the unit cell as a function of the time. The kinetic energy more than $0.12 \mathrm{eV}$ is concentrated solely on $\mathrm{C}_{3}$ atom in the direction inward the vacancy. The kinetic energy distribution on each atom thus shows very athermal property of the system. So far, the atom $\mathrm{C}_{3}$ is still holding its direction and we are not able to conclude whether this kind of atomic motion show a reversible motion (vibration) or results in decay of the CNT.

\section{Single vacancy with self-stabilization}

We finally show another shape of a single vacancy, which can be seen in narrow CNTs. Figure 6 shows relaxed structure without restriction of the mirror symmetry. Two of the three atoms adjacent to the vacancy, for instance $\mathrm{C}_{2}$ and $\mathrm{C}_{3}$ in the $(3,3)$ nanotube, form a dimmer and the other atom has a dangling bond. We have checked that this kind of dimerization never occurs in a single vacancy of planar graphene sheet. (According to a calculation with use of DFT supercell band-structure theory[17], the single vacancy on a graphene sheet shows the Jahn-Teller type distortion, which is, however, not large enough to show the dimerization in two of the three atoms adjacent to the vacancy.) The energy gain from non-dimerized structure to the dimerized structure is dependent on tube diameter. The value of the gain is $1.76 \mathrm{eV}$ for $(3,3)$ nanotube, $1.53 \mathrm{eV}$ for $(5,5)$ nanotube, and $1.23 \mathrm{eV}$ for $(7,7)$ nanotube, according to the present LDA calculations. Meanwhile, the C-C bond lengths of the dimer increases as the diameter increases: $1.52 \AA$ for $(3,3)$ nanotube, $1.56 \AA$ for $(5,5)$ nanotube, and $1.61 \AA$ for $(7,7)$ nanotube. It seems that there is an activation barrier from non-dimerized structure to the dimerized structure and that the value also depends on tube diameter. However, we have not determined the value yet.

The present data show a huge energy gain beyond $1 \mathrm{eV}$ upon dimerization in all of the investigated CNTs, so we expect that the vacancy in the $(10,10)$ nanotube should also show similar dimerization. If this is the case, the I-V curve shows disappearance of some of the sharp dips originating from the 
defect related states[5] because the dimerization pushes the corresponding energy levels far from $\mathrm{E}_{\mathrm{F}}$. Since energy gain upon the dimerization increases with decreasing tube diameters, we conclude that the dimerization is aided by a curvature of tube wall, and will not be seen in large diameter CNTs.

\section{Concluding remarks}

We have performed theoretical test for the toughness of defected CNTs focusing on a single vacancy. According to the Tight-Binding MD for the defected $(3,3)$ and $(4,4)$ nanotubes, no indication of the decay of the tubes were observed up to $4000 \mathrm{~K}$ in the simulated temperature. It is surprising that such narrow CNTs can retain its cylindrical structure despite a huge strain energy originating from the curvature of tube walls. Yet we must keep in mind that the simulation time is too short to conclude that no melting occurs.

Electronic excitation with a low energy (up to $1 \mathrm{eV}$ ) can induce lattice motion with long lifetime. Since initial excitation increases anti-bonding nature in two of the three atoms adjacent to the defects, these two atoms start to show a vibration. Meanwhile the other atom showed irreversible motion, so far, as to go inside the vacancy. It is not yet able to conclude whether this motion shows a vibration or turns out to be collapse of this CNT. This simulation is still running and the results will be presented in elsewhere.

The partial dimerization of two of the three atoms adjacent to a single vacancy might be a promising sign of CNTs for device applications. This kind of dimerization can increase mechanical toughness and can decrease the density of state near $\mathrm{E}_{\mathrm{F}}$, so that the property of a defected CNT becomes closer to that of the perfect CNT. Such a reduction of the density of state near $\mathrm{E}_{\mathrm{F}}$ upon dimerization is expected to suppress the ionic motion induced by low-energy electronic excitation. We thus expect that narrower nanotube are tolerant to presence of vacancies in applying to the electronic devices. We have also observed a dimerization in a single vacancy of zigzag CNTs the details will be presented in elsewhere.

\section{References}

[1] S. Iijima, Nature (London) 354, 56 (1991).

[2] M. Kosaka, T. W. Ebbessen, H. Hiura, and K. Tanigaki, Chem Phys. Lett. 233, 47 (1995). 
[3] F. Beubeu abd C. I'Huillier, J. -P. Salvetat, J. -M. Bonard, and . Forró, Phys. Rev. B59, 5945 (1999).

[4] M. Igami, T. Nakanishi, and T. Ando, J. Phys. Soc. Jpn. 68, 716 (1998).

[5] H. J. Choi, J. S. Ihm, S. G. Louie, and M. L. Cohen, Phys. Rev. Lett. 84, 2917 (2000).

[6] See, for example, http://www.research.ibm.com/resources/press/logiccircuit/

[7] L. C. Qin, X. Zhao, K. Hirahara, Y. Miyamoto, Y. Ando, and S. Iijima, Nature (London) 408, 50 (2000).

[8] N. Wang, Z. K. Tang, G. D. Li, and J. S. Chen, Nature (London) 408, 50 (2000).

[9] J. Ihm, A. Zunger, and M. L. Cohen, J. Phys. C 12, 4409 (1979).

[10] J. P. Perdew and A. Zunger, Phys. Rev. B23, 5048 (1981).

[11] D. M. Ceperley and B. J. Alder, Phys. Rev. Lett., 45, 566 (1980).

[12] N. Troullier and J. L. Martins, Phys. Rev. B43,1993 (1991).

[13] L. Kleinmann and D. M. Bylander, Phys. Rev. Lett. 48, 1425 (1982).

[14] Y. K. Kwon and D. Tománek, Phys. Rev. B58, R16001 (1998).

[15] O. Sugino and Y. Miyamoto, Phys. Rev. B.59, 2579 (1999).

[16] M. Suzuki, J. Phys. Soc. Jpn., 61, L3015 (1992).

[17] M. Saito in private communication. 
Fig. 1. Atomic geometry for single vacancies in $(n, n)$ nanotubes, where (a) $n=3$, (b) $n=5$, and (c) $n=7$. Hatched and filled circles denote carbon atoms being apart from and adjacent to the vacancy, respectively. The displayed geometry corresponds to twice of the present unit cell.

Fig. 2. Results of the present TBMD simulations for a $(3,3)$ nanotube with an array of single vacancies. (a) History of temperature as a function of simulation time, which was raised by 400 degrees at every simulation period of $0.75 \mathrm{ps}$. (b) The final snap shot of the TBMD simulation, in which the survival of a cylindrical shape can be seen.

Fig. 3. Single-electron energy levels of the $(3,3)$ nanotube with a single vacancy at every unit cell having quadruple period along the tube axis of the primitive cell of the armchair CNT. Bars are the energy level under electronic ground state (the left column) and under the electronic excited state (the right column). Insets are contour map of norm of each eigen functions related with eigen values by arrows. 
Fig. 4. Time evolution of single-electron energy levels throughout the electron-ion dynamics simulation of the $(3,3)$ nanotube with presence of a single vacancy. Thin solid and dotted lines are fully occupied and empty states, while thick dotted lines are half occupied states representing a hole (lower line) and an excited electron (upper line).

Fig. 5. (a) Atomic positions before and $70 \mathrm{fs}$ after the electron-ion dynamics. Atoms adjacent to the defects are labeled by $\mathrm{C}_{1}, \mathrm{C}_{2}$, and $\mathrm{C}_{3}$. (b) Distribution of the kinetic energies on all atoms per unit cell as a function of the time. The corresponding atomic labels are also denoted.

Fig. 6. Atomic geometry of C-C dimer seen in single vacancies of CNT for (a) $(3,3)$, (b) $(5,5)$, and (c) $(7,7)$ nanotubes. Solid circles denote carbon atoms adjacent to the vacancies, and the dimer bonds are shown as black sticks. 

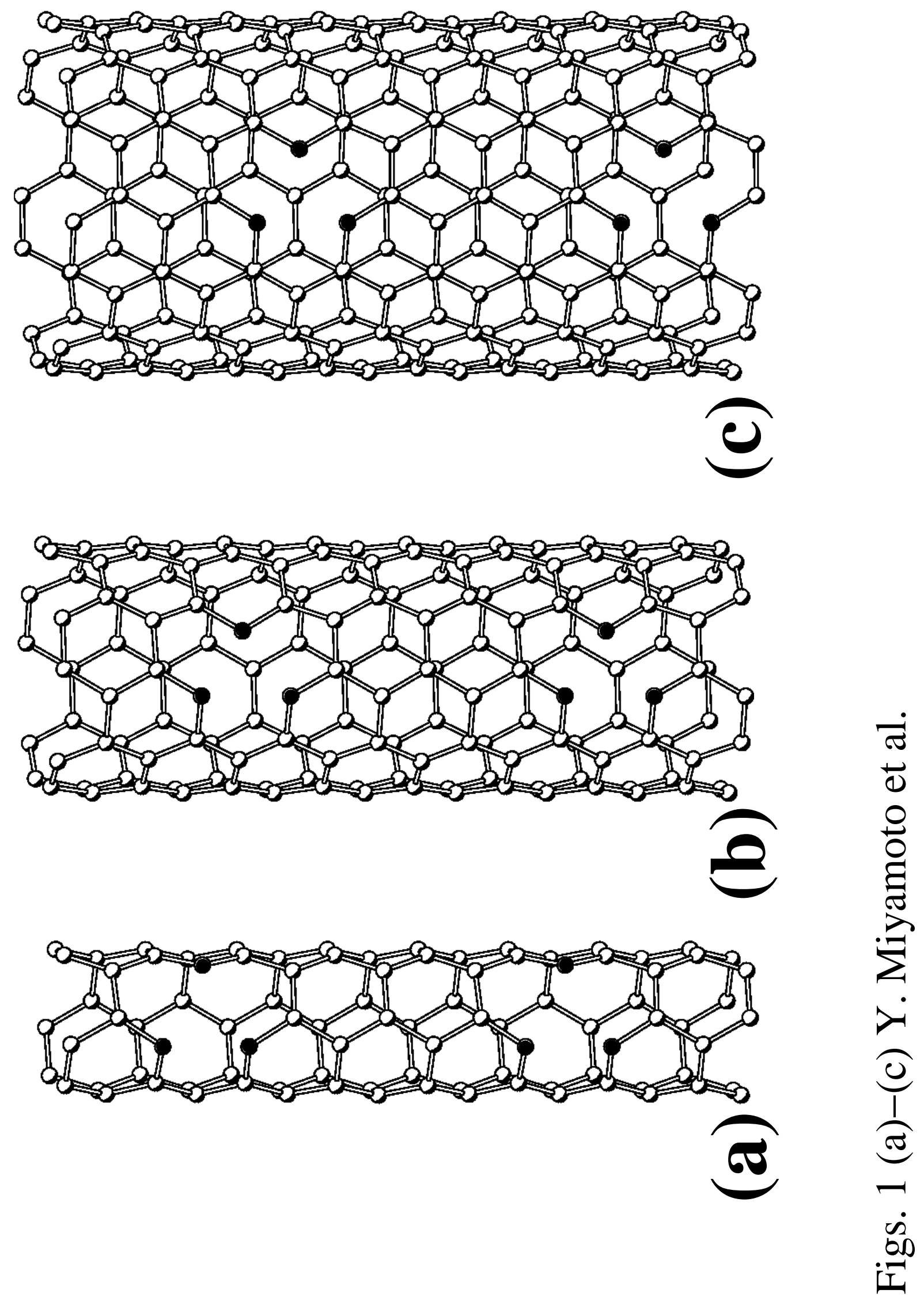

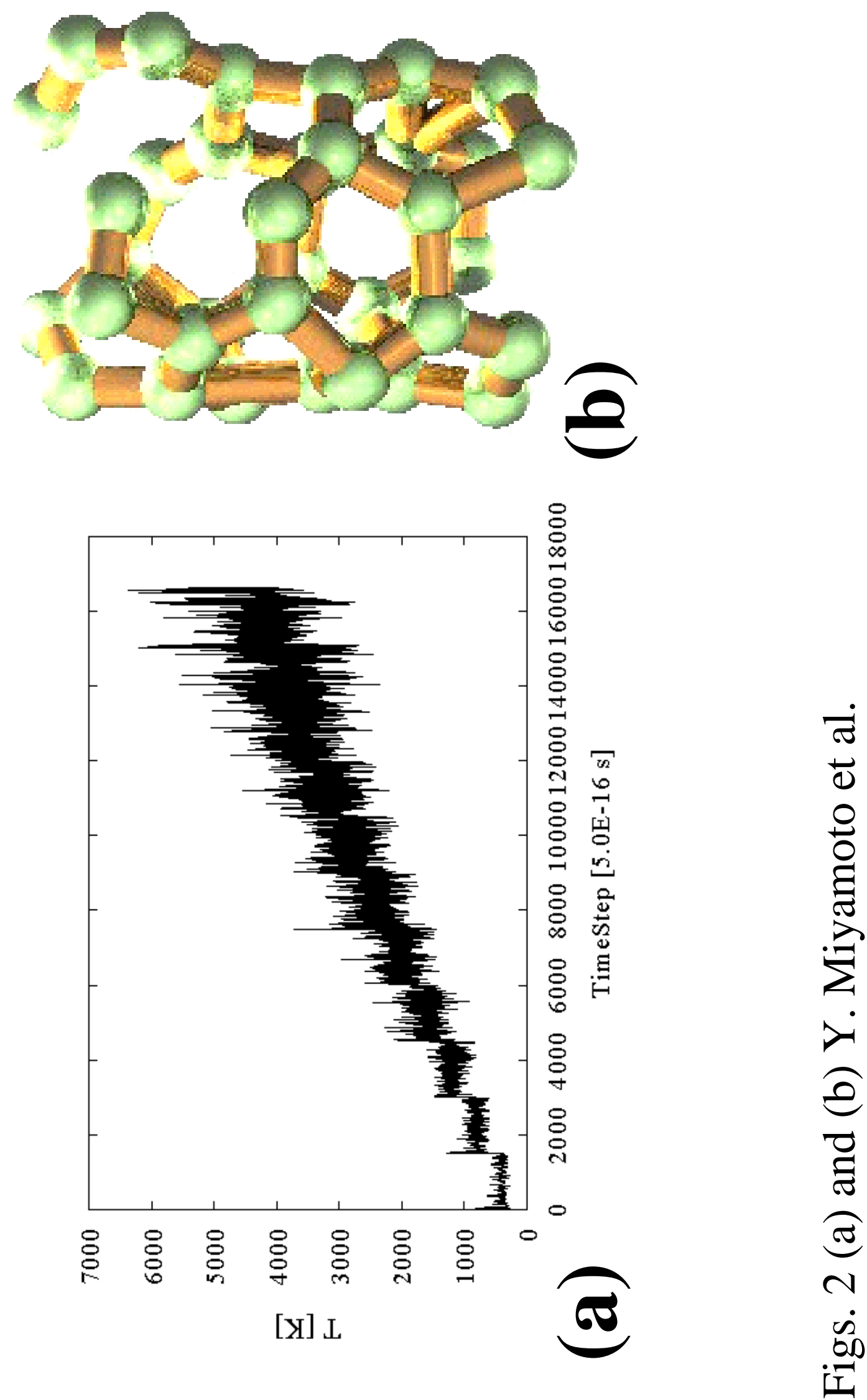


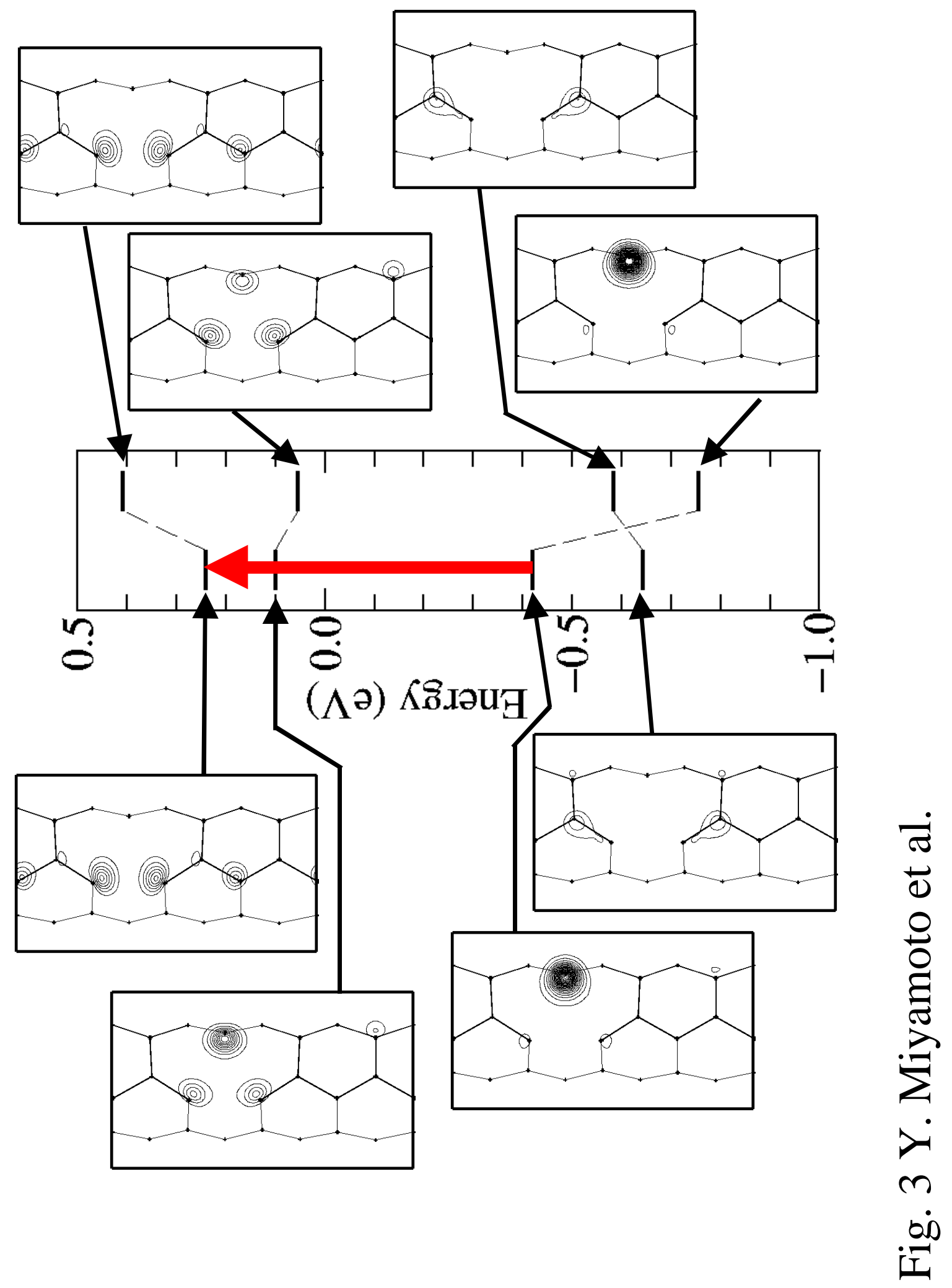



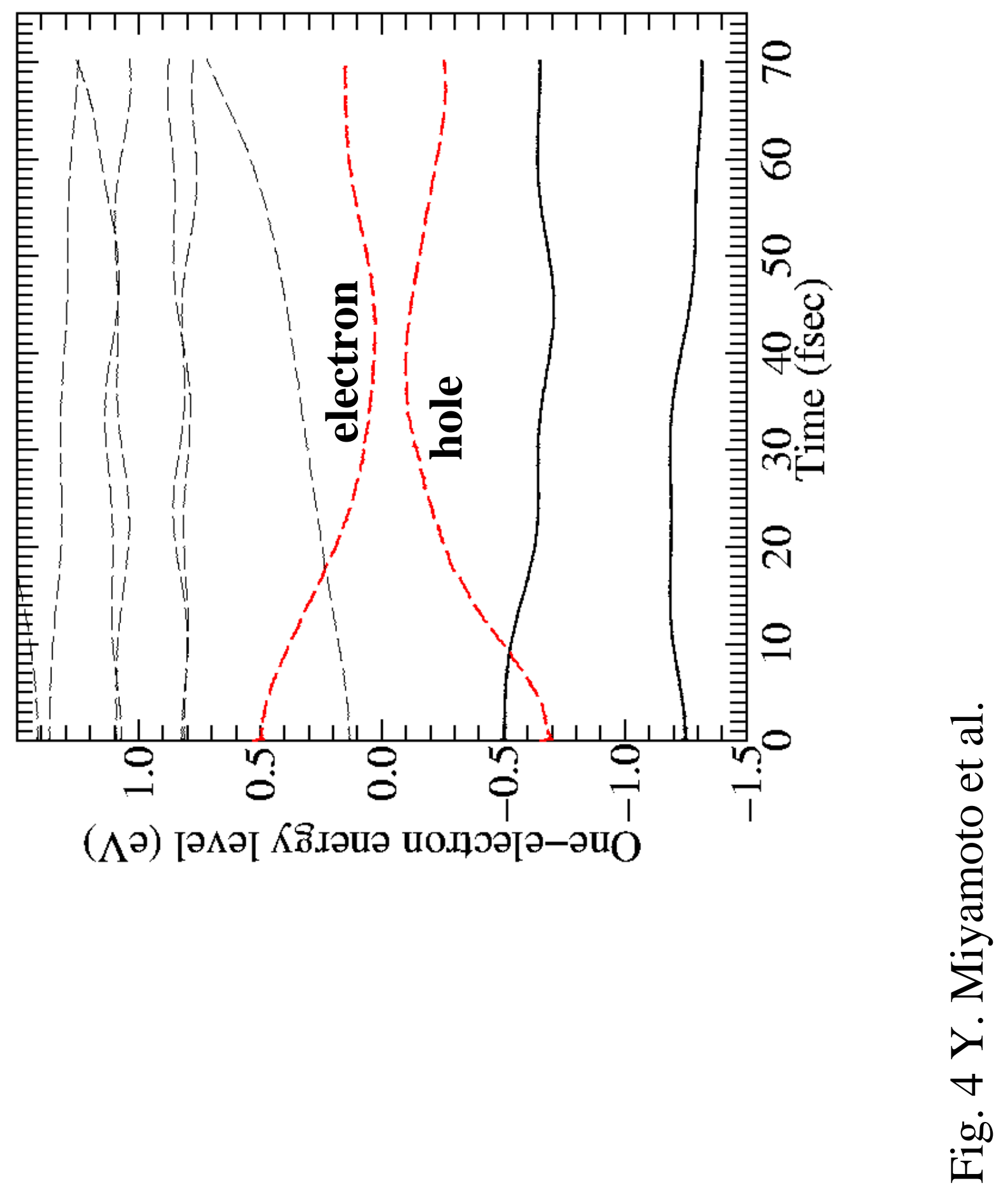

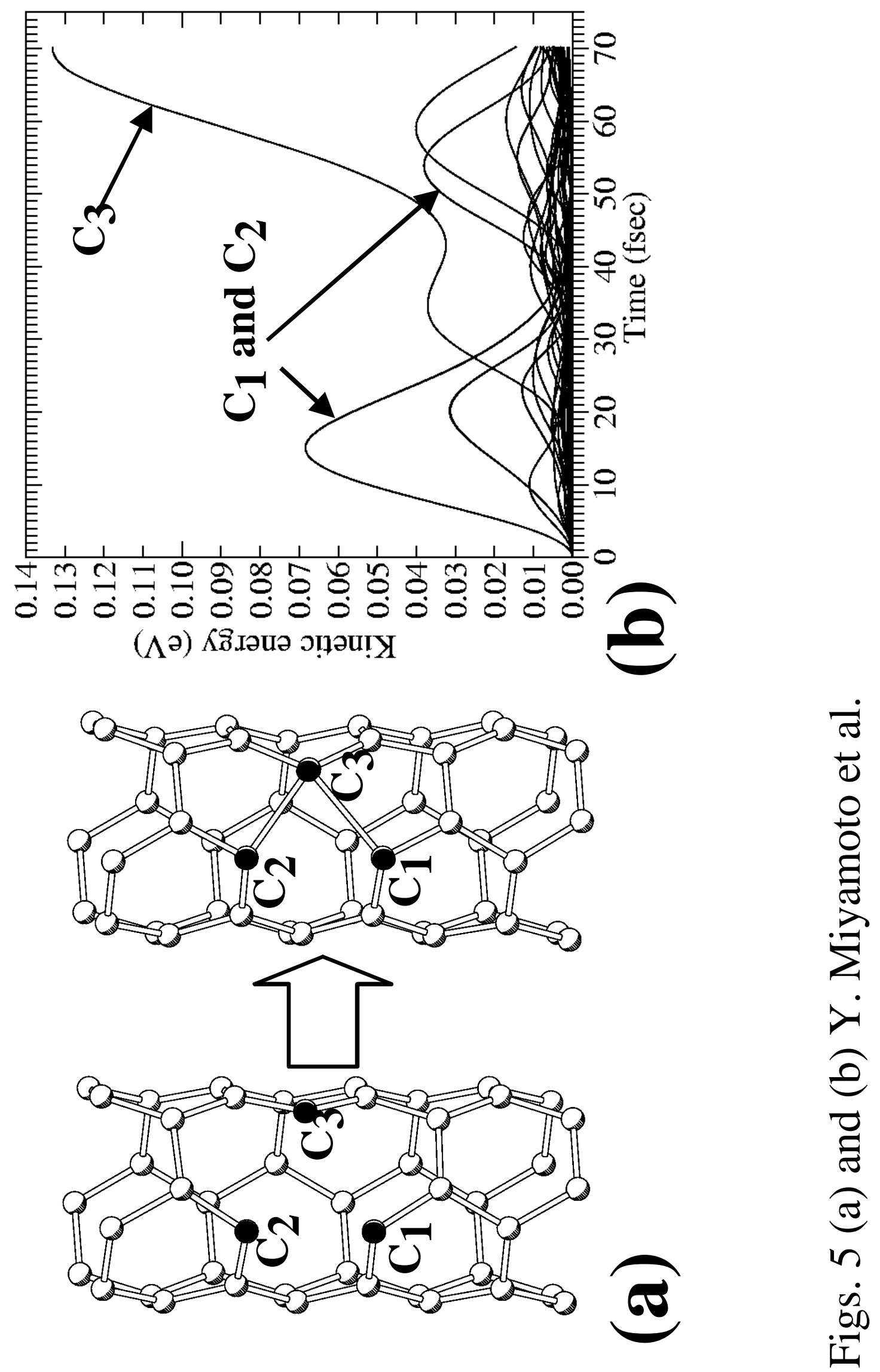

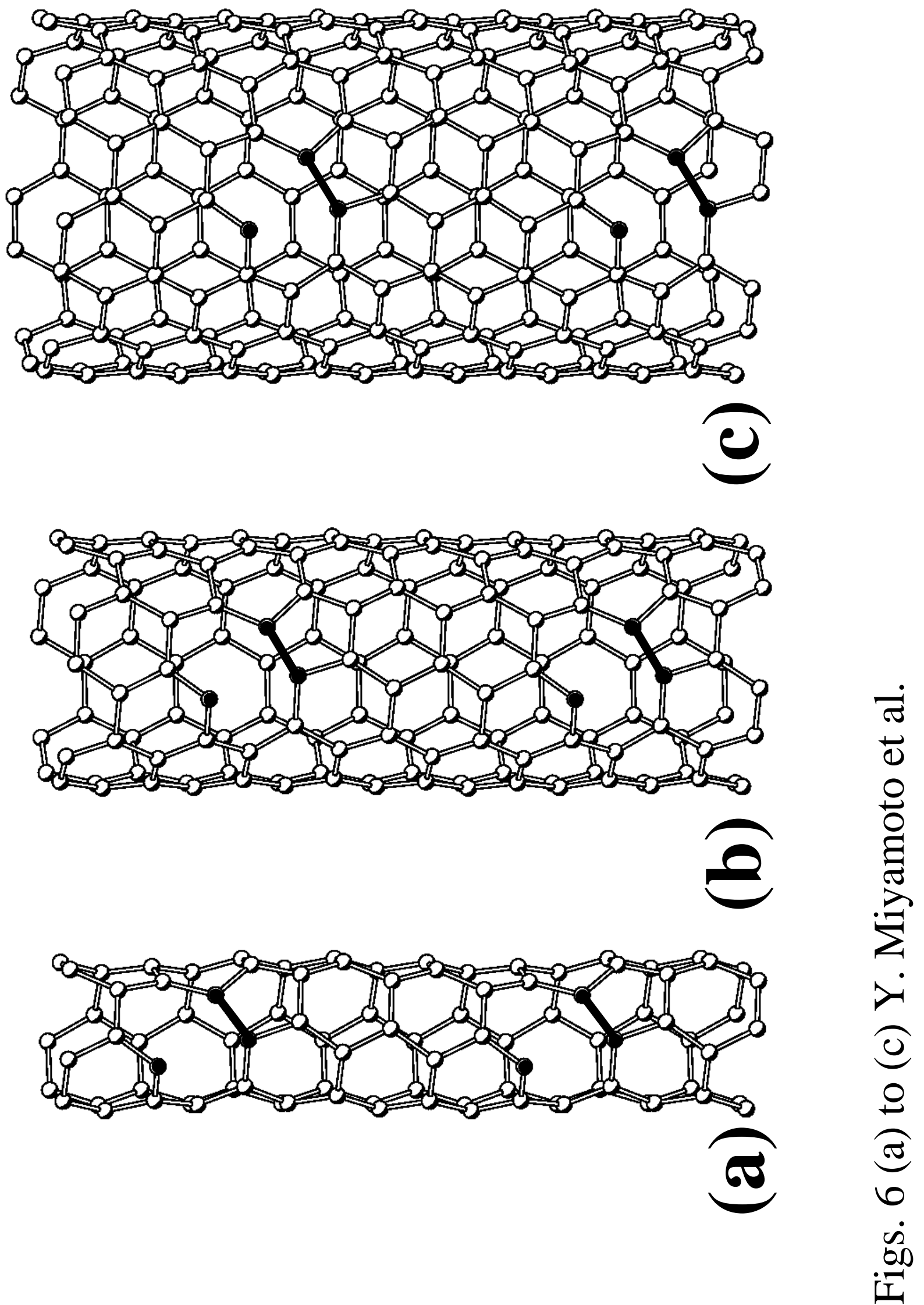